\title{
Recurrent Challenges for Clinicians: Emergence of Methicillin-resistant Staphylococcus aureus, Vancomycin Resistance, and Current Treatment Options
}

\section{Bansidhar Tarai, Poonam Das, Dilip Kumar}

Department of Microbiology, Max Super Speciality Hospital, Saket, New Delhi, India

Address for correspondence: Dr. Bansidhar Tarai, E-mail: bansisss@gmail.com

\section{ABSTRACT}

Gram-positive pathogens mainly, Staphylococcus aureus, Enterococcus and coagulase-negative Staphylococcus, are developing increasing resistance to glycopeptides that pose a problem in treating infections caused by these pathogens. Vancomycin is the treatment of choice in treating methicillin-resistant $S$. aureus (MRSA). Community-acquired MRSA is associated with infections in patients without recent history of hospital admission and without the classical risk factors for MRSA carriage (including healthcare personnel). MRSA poses new threats and challenges beyond the hospital with the emergence of community-acquired MRSA. Indiscriminate use of vancomycin leads to the emergence and spread of vancomycin resistance in multidrug resistant strains is of growing concern in the recent years. Minimum Inhibitory concentration (MIC) remains an important determinant in choosing the right antibiotics. Infections caused by MRSA strains with vancomycin MIC $>4 \mu \mathrm{g} / \mathrm{mL}$ leads to the vancomycin treatment failure. The Clinical Laboratory Standards Institute had also lowered the cut-off susceptibility and resistance breakpoints for vancomycin. Despite the availability of newer antimicrobial agents (Linezolid, Daptomycin, Tigecycline) for drug-resistant Gram-positive pathogens, clinicians and patients still need options for treatment of MRSA infection. There is a need to reduce the global burden of infections caused by Gram-positive pathogens and its resistant strains (mainly MRSA). Continuous efforts should be made to prevent the spread and the emergence of glycopeptide resistance by early detection of the resistant strains and using the proper infection control measures in the hospital setting.

Key words: Enterococcus, Gram-positive, minimum inhibitory concentration, methicillin resistant Staphylococcus aureas, resistance, Staphylococcus aureus, vancomycin, vancomycin-intermediate Staphylococcus aureus

\section{INTRODUCTION}

\% loodstream infection (BSI) is defined as infections caused by bacteria in the bloodstream with signs and/or symptoms of infection. ${ }^{[1]}$ BSIs are usually serious infections, that can prolong hospitalization and increase hospitalization costs and risk of mortality. Staphylococcus aureus (S. aureus) is one of the most common causes of BSI, skin and wound infections, osteomyelitis, endocarditis,

\begin{tabular}{|l|l|}
\hline \multicolumn{3}{c}{ Access this article online } \\
\hline Quick Response Code: & Website: \\
&
\end{tabular}

and nosocomial infections, especially pneumonia, surgical site infections, and continue to be a major cause of community-acquired infections. ${ }^{[2,3]}$ Several epidemiologic studies have demonstrated that infection due to $S$. aureus is associated with increased burden on healthcare resources and increased morbidity and mortality. ${ }^{[1]}$ The development of antimicrobial resistance has been regarded as a consequence of their use since their introduction nearly 70 years ago and is continuously worsening. ${ }^{[4]}$

Methicillin-resistant $S$. aureus (MRSA) is a major cause of nosocomial and community acquired infections ${ }^{\left[{ }^{[}\right]}$ and continue to cause a variety of clinical syndromes worldwide. ${ }^{[3]}$ There are a few number of newer antimicrobial agents available to treat these lethal infections. ${ }^{[1]}$ In Indian hospitals, based on antibiotic sensitivity tests, 30 to $80 \%$ MRSA, has been reported. ${ }^{[2]}$ 
Glycopeptides are widely used for the prophylaxis and treatment of various gram-positive infections. Vancomycin once considered the gold standard for the treatment of multidrug resistant (MDR) $S$. aureus, and is increasingly being used to treat other infections, such as pseudomembranous colitis due to Clostridium difficile and coagulase-negative staphylococci (CoNS) infections in hospitalized patients. ${ }^{[6]}$ Use of vancomycin extensively can create a selection pressure favoring the development of rare, vancomycin-resistant strains. These strains will lead to the emergence of heterogeneous vancomycin intermediate S. aureus (hVISA) strains, and eventually, with continued exposure, to a uniform population of VISA strains. For the last 7 years, incidence of VISA and vancomycin-resistant S. aureus (VRSA) has been increasing in various parts of the world. ${ }^{[7]} S$. aureus causes life-threatening infections in hospitalized and non-hospitalized patients. ${ }^{[6]}$ Increase in the prevalence of vancomycin resistant strains is a challenging and serious public health concern.

Vancomycin-resistant $S$. aureus tends to be multi drug resistant against a large number of currently available antimicrobial agents, compromising treatment options and increasing the likelihood of inadequate antimicrobial therapy and increase in morbidity and mortality. The emergence of VRSA is a critical concern to the therapeutic dilemma caused by the presence of multi drug resistant organisms in recent years. ${ }^{[6]}$

Increasing minimum inhibitory concentration (MIC) of Vancomycin over the time, though within the susceptible range, (Vancomycin MIC creep) could be associated with vancomycin treatment failures. Higher MICs are correlated with, administration of higher doses to achieve target drug levels, lower success rates and higher mortality rates. Years of exposure of strains to sub-inhibitory concentrations of vancomycin is thought to be a key factor for increasing vancomycin MICs. For an individual patient, prior exposure to vancomycin appears to be important. ${ }^{[3]}$ The previous breakpoints for vancomycin have been revised from $(<4 \mu \mathrm{g} / \mathrm{mL}[\mathrm{S}] ; 8-16 \mu \mathrm{g} / \mathrm{mL}[\mathrm{I}] ;>32 \mu \mathrm{g} / \mathrm{mL}[\mathrm{R}])$ to $(<2 \mu \mathrm{g} / \mathrm{mL}[\mathrm{S}] ; 4-8 \mu \mathrm{g} / \mathrm{mL}[\mathrm{I}] ;>16 \mu \mathrm{g} / \mathrm{mL}[\mathrm{R}])$. $^{[2,3]} \mathrm{In}$ Japan, however, isolates with MIC $8 \mu \mathrm{g} / \mathrm{mL}$ are considered VRSA. ${ }^{[6]}$

From India, there are reports of rise in resistance to the antibiotics such as penicillin, semisynthetic penicillin (methicillin, oxacillin, etc.), macrolides, tetracycline, and aminoglycosides. ${ }^{[5]}$ Reasons for the development of antibiotic resistance in developing countries (e.g., India) could be the irrational antibiotic usage. The factors contributing to this are, easy availability of antibiotics at the drug store without prescription, extensive surgical procedures, lack of barrier nursing practices, prolonged hospitalization, and indiscriminate use of antibiotics in hospitals, agriculture, animal husbandry, and fisheries. ${ }^{[8]}$

The emergence and spread of resistance to vancomycin is a threat to the already challenging therapy of MRSA and raise an alarming situation to the clinicians in hospital as well as in community. ${ }^{[8]}$ The spread of MRSA from the hospital to the community, coupled with the emergence of VISA and VRSA, have become a major concern among healthcare providers. ${ }^{[6]}$ Numerous challenges were faced by scientists to develop new therapies targeting drug-resistant pathogens. ${ }^{[1]}$ Antimicrobial resistance to penicillin, methicillin, or vancomycin is an unavoidable consequence of the selective pressure of antibiotic exposure. Minimizing the antibiotic pressure is essential to control the emergence of resistant strains in the hospital and in the community. ${ }^{[8]}$

The aim of this article is to understand the emergence of the BSI caused by MRSA, VRSA, VISA, and vancomycin-resistant Enterococci (VRE) and to evaluate the emerging challenges to the clinicians in treating these infections.

\section{METHICILLIN-RESISTANT STAPHYLOCOCCUS AUREUS}

\section{Epidemiology}

Despite the recent reports that gram-negative bacteria have overtaken staphylococci as the leading cause of nosocomial infections, MRSA continues to be a threat in the health care settings. ${ }^{[8]}$

MRSA can be defined as the strains of $S$. aureus that are resistant to the isoxazoyl penicillins such as methicillin, oxacillin and flucloxacillin. MRSA offers cross-resistance to all currently licensed $\beta$-lactam antibiotics. ${ }^{\left[{ }^{[6}\right.}$ Methicillin resistance arises through the production of a supplementary penicillin-binding protein (PBP), known as PBP2' or PBP2a. This compensates for the functions of the essential methicillin-susceptible staphylococcal PBPs and confers resistance to most $\beta$-lactams. ${ }^{\left[{ }^{[0]}\right.}$

MRSA was first reported in 1961 and over the last 5 decades, it still continues to be clinically important pathogen. ${ }^{[2]}$ Since the late 1990s, an increasing number of cases of community-acquired MRSA have been reported worldwide including the USA, Europe, New Zealand, Australia, Samoa, Canada, and Finland, to the extent that it is being considered in some parts of the world to be epidemic. $^{[4]}$ 
Infections caused by MRSA have been associated with high morbidity and mortality rates. The prevalence of methicillin-resistant strains varies globally from 20\% in Europe ${ }^{[10]} 33-55 \%$ in the United States ${ }^{[10]}$ and 30 to $80 \%$ in India. ${ }^{[5]}$ In Indian hospitals, MRSA is one of the common cause of hospital-acquired infections and different hospitals have reported methicillin resistance based on antibiotic sensitivity tests. ${ }^{[5]}$ In India, prevalence of community MRSA nasal colonization has been reported to be $4 \%$ in children of $5-15$ years of age.

In addition to being methicillin resistant, most MRSA strains are also resistant to other $\beta$-lactam antibiotic, with the exception of glycopeptide antibiotics. In 1980s, because of widespread occurrence of MRSA, the empiric therapy for staphylococcal infections (particularly nosocomial sepsis) was changed to vancomycin in many health care institutions. ${ }^{[7]}$

\section{Correct approach for resistance detection}

The Clinical and Laboratory Standards Institute (CLSI) recommends the cefoxitin-disk $(30 \mu \mathrm{g})$ screen test, the latex agglutination test for PBP2a, or a plate containing $6 \mu \mathrm{g} / \mathrm{mL}$ of oxacillin, in Mueller-Hinton agar supplemented with $\mathrm{NaCl}(4 \% \mathrm{w} / \mathrm{v} ; 0.68 \mathrm{~mol} / \mathrm{L})$ as alternative methods of testing for MRSA and mecA detection; based on PCR or hybridization. ${ }^{[6]}$ Disk-diffusion method is reliable if incubation temperature is maintained at $35^{\circ} \mathrm{C}$ for 24 hours. Accurate detection of oxacillin/methicillin resistance can be difficult due to the presence of 2 subpopulations (one susceptible and the other resistant) that may coexist within a culture of staphylococci, i.e. they are heteroresistant. ${ }^{[6]}$

In a study by Tiwari and Sen, ${ }^{[7]}$ a of total 1681 staphylococcal isolates consisting of $783 \mathrm{~S}$. aureus and $898 \mathrm{CoNS}$ were isolated from different clinical specimens from various outpatient departments and wards. All S. aureus and 93 CoNS were subjected to MIC testing (against vancomycin, teicolplanin and oxacillin); Brain Heart Infusion (BHI) vancomycin screen agar test; disc diffusion testing, and PCR for mec $A$, van $A$ and $v a n B$ genes detection. Out of 783 S. aureus two $S$. aureus strains were found to be vancomycin and teicoplanin resistant (one strain with MIC $32 \mu \mathrm{g} / \mathrm{ml}$ and the other strain with MIC $64 \mu \mathrm{g} / \mathrm{ml}$ ); six strains of $S$. aureus have shown to be vancomycin intermediate (two strains with MIC $16 \mu \mathrm{g} / \mathrm{ml}$ and four strains with MIC $8 \mu \mathrm{g} / \mathrm{ml}$ ); and two strains with teicoplanin intermediate (MIC $16 \mu \mathrm{g} / \mathrm{ml}$ ). 1 CoNS strainwas resistant to vancomycin and teicoplanin (MIC $32 \mu \mathrm{g} / \mathrm{ml}$ ), and two CoNS strains were intermediate to vancomycin and teicoplanin (MIC $16 \mu \mathrm{g} / \mathrm{ml}$ ). All VRSA, VISA and vancomycin resistant CoNS had shown growth on BHI vancomycin screen agar (vancomycin $6 \mu \mathrm{g} / \mathrm{ml}$ ) and were mec $A$ PCR positive. None of these isolates have demonstrated van $A /$ vanB gene by PCR.

The standard protocol is being followed at our Hospital for screening of sepsis. ${ }^{[11,12]}$ The following tests are conducted for screening of sepsis e.g., Paired blood culture tests, procalcitonin and C-reactive protein (CRP) and for screening of sepsis in Intensive care unit (ICU); Paired blood culture test, procalcitonin, CRP, Endotracheal grams stain, Endotracheal culture and sensitivity, urine routine and microscopy, and urine culture and sensitivity, are done routinely.

At our hospital, paired and single blood cultures were processed in BacT/ALERT three dimensional blood culture system (Biomerieux). Rests of the samples were processed aseptically inside the Biosafety Cabinet. The gram-positive pathogens were identified in VITEK 2 Compact and susceptibility and MIC done in Vitek 2 and E-strip (Biomerieux).

In a study by Dhanalakshmi et al., a total of 250 non-duplicate isolates of $S$. aureus were isolated and tested for methicillin resistance by using the oxacillin disc diffusion test $(1 \mu \mathrm{g})$, the cefoxitin disc diffusion test $(30 \mu \mathrm{g})$ and the oxacillin agar screen method (Muller Hinton agar with $4 \% \mathrm{NaCl}^{+} 6 \mu \mathrm{g} / \mathrm{ml}$ oxacillin). Out of the 250 isolates, $80(32 \%)$ isolates were found to be methicillin resistant by the cefoxitin disc diffusion method, 78 (31.2\%) were methicillin resistant by the oxacillin agar screen test and $77(30.8 \%)$ were methicillin resistant by the oxacillin disc diffusion method. Sixty seven $(83.8 \%)$ of the 80 MRSA isolates and $26.8 \%$ of the total $250 \mathrm{~S}$. aureus isolates tested were found to be MDR-MRSA. ${ }^{[13]}$

\section{Clinical importance and therapeutic measures}

A single positive blood culture for $S$. aureus should prompt an initiation of empirical antibiotic therapy and aggressive surgical therapy of any drainable foci of infection ${ }^{[1]}$ MRSA acquired during exposure in hospitals and other healthcare facilities causes a variety of serious healthcare-associated infections. The problem is exacerbated by the propensity of the organism to cause cross-infection and its ability to colonize individuals for months or years. ${ }^{[6]}$

MRSA-colonized patients more frequently develop symptomatic infections than Methicillin Sensitive Staphylococcus aureas-colonized patients do. Once MRSA is introduced into a healthcare setting, transmission and persistence of the resistant strain is determined by the 
availability of vulnerable patients, selective pressure exerted by antimicrobial use, increased potential for transmission from larger numbers of colonized or infected patients ("colonization pressure"), and the impact of implementation and adherence to prevention efforts. ${ }^{[6]}$ Patients vulnerable to colonization and infection include those with severe disease, especially those with compromised host defenses from underlying medical conditions; recent surgery; or indwelling medical devices (e.g., urinary catheters or endotracheal tubes). ${ }^{[6]}$ Hospitalized patients, especially ICU patients, tend to have more risk factors than non-hospitalized patients and have the highest infection rates. The prevalence of MRSA colonization and infection in the surrounding community may therefore affect the selection of strategies for MRSA control in healthcare settings. ${ }^{[6]}$

Therapeutic measures include improvements in hand hygiene, use of contact precautions until patients are culture-negative for MRSA, active surveillance cultures, education, enhanced environmental cleaning and improvements in communication about patients with MRSA, within and between healthcare facilities. ${ }^{[6]}$

Drugs approved for the treatment of MRSA infections are vancomycin, linezolid, daptomycin, teicoplanin, quinupristine-dalfopristine and tigecycline. Vancomycin is the drug of choice for therapy of infections due to MRSA.

\section{VANCOMYCIN}

Vancomycin was available for clinical use after its approval by Food and Drug Administration in 1958. The introduction of vancomycin as an anti-staphylococcal agent was followed shortly thereafter by methicillin, the cephalosporin's, and the linomycins, drugs which initially received wider clinical acceptance in contrast to vancomycin as a consequence of the apparent toxic side effects of the latter. ${ }^{[14]}$

The increase in vancomycin resistance among MRSA and MDR-MRSA and excessive use of antimicrobial agents has worsened the sensitivity, which call for further epidemiological studies. ${ }^{[2,13]}$ Glycopeptide resistance was first detected in the mid-1980s among clinical isolates of gram-positive bacteria. Since 1996, five VISA strains have been identified in Europe, Asia, and United States, and VRSA strains have also been reported in the United States between 2002 and 2005. Most infections with VISA and VRSA have occurred in a setting of substantial prior use of glycopeptides and other antimicrobial agents. ${ }^{[10]}$

\section{Mechanism of vancomycin resistance}

The emergence of VRSA strains exhibits two different resistant mechanisms: (1) Thickened and poorly cross-linked cell wall, whereby many vancomycin molecules are trapped within the cell wall. Biochemical and transmission electron microscopy examination of the Mu50 cell, suggested that it produces increased amounts of peptidoglycan. ${ }^{[7]}$ More murein monomers and more layers of peptidoglycan is considered to be present in the cell wall. ${ }^{[7]}$ The confined molecules then block the peptidoglycan meshwork and finally form a physical barrier towards further incoming vancomycin molecules (VISA noted in Japan 1996 and subsequently in United States in 1997); (2) Mechanism is identical as observed in VRE, i.e. inhibiting the incorporation of ( $\mathrm{N}$-acetylmuramic acid - $\mathrm{N}$-acetylglucosamine) into the growing peptidoglycan chain by reacting with $\mathrm{D}$-Ala-DAla which consequently blocks the release of terminal D-Ala, and intrachain bond formation (Noted in United States in 2002). ${ }^{[5]}$ Subsequent isolation of VISA and VRSA isolates from other countries including Brazil, France, United Kingdom, Germany, India, and Belgium has confirmed that the emergence of these strains is a global issue. ${ }^{[2]}$

\section{Correct approach for resistance detection}

VRSA isolates are detected by reference broth microdilution, agar dilution, E-test ${ }^{\circledR}$, MicroScan $^{\circledR}$ overnight, BD Phoenix ${ }^{\mathrm{TM}}$ system, VRSA screen test for VITEK ${ }^{\circledR} 2$, Synergies plus ${ }^{\mathrm{T} M}$, TREK Sensititre MIC plate, disk diffusion and vancomycin screen agar plates (brain-heart infusion agar containing $6 \mu \mathrm{g} / \mathrm{L}$ vancomycin). Two acceptable primary test methods are (a) MIC method plus vancomycin VA screen plate and (b) disk diffusion and VA screen plate. Based on this, possible VISA and VRSA strains are identified. ${ }^{[6]}$ The clinical microbiology laboratories must ensure using detection methods with good sensitivity and specificity. More studies are needed for finding the accurate screening method for VISA. ${ }^{[13]}$

Vancomycin-resistant strains (VISA, VRSA, and VRE)

Patients at the risk of developing VISA and VRSA are the ones with previous exposure to vancomycin. Reduced susceptibility to vancomycin is seen in the elderly and in those with chronic wounds or decubitus ulcers mainly infected with VRE, which were probably poor donor organisms of the vanA gene to $S$. aureus. ${ }^{[8]}$

It has been suggested that patients at risk for VRSA are co-infected or co-colonized with VRE and MRSA, which 
enables transfer of vanA gene from VRE to MRSA in a biofilm environment leading to a VRSA strain. ${ }^{[6]}$

The first case of hVISA was reported in Japan in 1997. ${ }^{[6]}$ The prevalence of hVISA was reported in the range of $0 \%$ to $74 \%$ in various countries, including Japan, Tehran, India, Korea, Hong Kong, Thailand, Spain, Greece, Germany, Italy, and the United Kingdom. ${ }^{[6]}$ Strains of VISA with vancomycin MIC of $8 \mu \mathrm{g} / \mathrm{ml}$ have been reported from Japan, United States, France, United Kingdom, and Germany. Most of these isolates appear to have developed from preexisting MRSA infections. ${ }^{[7]}$

Enterococcal infections are more common in elderly patients due to various associated risk factors. Infections due to VRE include urinary tract, wound infections, BSI, endocarditis, and meningitis. Antibiotic resistance among enterococci is conferred through mutation and acquisition of genetic material from other species. ${ }^{[3]}$

VRE was first reported in Europe in 1988 and later in New York City in 1989. Infection with VRE affects patients in ICU and those with intravascular or bladder catheter devices. ${ }^{[3]}$ Immunosuppressed patients, particularly recipients of liver and other solid organ transplants and hematopoietic stem cell transplants, remain vulnerable to VRE infections. Prolonged hospitalization, residence in long-term care facilities, and exposure to antibiotics are a cause factor for VRE infections. ${ }^{[3]}$ VRE bacteremia increases the length of hospital stay by an average of 2 weeks and mortality may exceed $50 \%$ in critically ill patients.

The CLSI defines VRE with an MIC of $32 \mu \mathrm{g} / \mathrm{mL}$ or more. ${ }^{[3]}$ A study comparatively evaluated paired blood culture (aerobic/aerobic) and single blood culture in 9364 clinical isolates. Of these, 680 isolates were found to be positive for bacteremia. Of 680 isolates, $69(10 \%)$ isolates were found to be gram-positive bacteria, 57 (8\%) were fungal and $554(82 \%)$ were gram-negative bacteria. True bacteremia was found in $12 \%$ isolates (6/52; MRSA 2, VRE 1, Enterococcus faecalis 1) of critical patients (paired blood culture). $\cdot^{11]}$

Clinical evidence of MRSA with reduced susceptibility to vancomycin

Vancomycin resistance has been a threat to the already challenging therapy of MRSA and MDR-MRSA. ${ }^{[13]}$ Various studies from India have reported the reduced susceptibility of vancomycin against MRSA and CoNS and recorded the emergence of low level and intermediate vancomycin resistance. Another study in the year 2004 has reported the emergence of heterogeneous VRSA strains from India and its neighboring countries.

The first report of VRSA emergence from a tertiary care hospital in North India was reported by Tiwari and Sen. ${ }^{[7]}$ They isolated 1681 staphylococcal isolates consisting of 783 S. aureus and 898 CoNS strains from different clinical specimens from various outpatient departments and wards of the University Hospital, BHU, Varanasi. This hospital serves the population of eastern region of Uttar Pradesh and adjoining districts of Bihar, Jharkhand, and Madhya Pradesh, India. Of 783 S. aureus, two S. aureus strains were found to be vancomycin and teicoplanin resistant (1 strain with MIC $32 \mu \mathrm{g} / \mathrm{mL}$ and the other strain with MIC $64 \mu \mathrm{g} / \mathrm{mL}$ ); six strains were VISA (2 strains with MIC $16 \mu \mathrm{g} / \mathrm{mL}$ and 4 strains with MIC $8 \mu \mathrm{g} / \mathrm{mL}$ ); and 2 strains with teicoplanin intermediate (MIC $16 \mu \mathrm{g} / \mathrm{mL}$ ). One CoNS strain was resistant to vancomycin and teicoplanin (MIC $32 \mu \mathrm{g} / \mathrm{mL}$ ), and 2 CoNS strains were intermediate to vancomycin and teicoplanin (MIC $16 \mu \mathrm{g} / \mathrm{mL}$ ). This study indicated the magnitude of antibiotic resistance in and around the study area, major cause being unawareness and indiscriminate use of broad-spectrum antibiotics. ${ }^{[7]}$

Thati et al. ${ }^{[2]}$ conducted a study in two tertiary care hospitals in Hyderabad, South India and observed that among the 358 clinical isolates of S. aureus, $285(79.6 \%)$ were identified as MRSA by disc diffusion method. Sixteen (4.4\%) isolates were identified as VISA (MIC $=4-8 \mathrm{mg} / \mathrm{L}$ ). All VRSA isolates ( $\mathrm{n}=7$ ) were MRSA and had a vancomycin MIC in the range of 16-64 mg/L. The VRSA isolates were positive for vanA gene, except one, which was negative.

Thorough care should be taken in the clinical microbiology laboratory while detecting the methicillin and vancomycin resistance, as there is only limited therapeutic alternatives available to treat the MRSA and the VRSA isolates. ${ }^{[13]}$

High MIC values of vancomycin among S. aureus Strains

The increase in vancomycin MIC could lead to the increase in the frequency of hetero-resistant VISA. Subpopulations of MRSA strains may have VISA selected by vancomycin treatment. Furthermore, increased vancomycin MIC has been correlated with adverse clinical outcomes in some studies. ${ }^{[3]}$

VISA and VRSA strains are not detected by the disk diffusion method. Acceptable methods used to detect these strains are non-automated and include broth or agar 
dilution and the E-test. The emergence of MRSA with vancomycin resistance and the magnitude of antibiotic resistance were observed in one study from south India. A total of 156 isolates from various clinical samples (pus, blood, urine, sputum, and body fluids) were collected from patients in India. All the MRSA isolates were uniformly sensitive to vancomycin by disc diffusion method. By agar dilution method, MIC of 18 isolates showed MIC of 4-8 $\mathrm{mcg} / \mathrm{mL}$ (VISA). No VRSA and hVISA were detected. Most of the VISA isolates were susceptible to amikacin, cotrimoxazole, ofloxacin, and resistant to other antibiotics. ${ }^{[8]}$

The emergence of vancomycin-resistant MRSA was also observed in Andhra Pradesh, India wherein among 84 clinical isolates of $S$. aureus, 67 isolates $(79.76 \%)$ were identified as MRSA and 3 isolates (3.57\%) were identified as vancomycin resistant by disc diffusion method. ${ }^{[5]}$ The MIC for 71 isolates $(84.52 \%$ ) was $\leq 2 \mathrm{mg} / \mathrm{L}$ (sensitive to vancomycin), for 10 isolates $(11.90 \%$ ) was $4-8 \mathrm{mg} / \mathrm{L}$ (VISA), and for 3 isolates (3.57\%), the MIC was in the range of 16-64 mg/L (VRSA). All three VRSA isolates were MRSA and showed resistance to a minimum of 7 antibiotics including vancomycin and methicillin. ${ }^{[5]}$ The occurrence of VRSA is further confirmed from another study ${ }^{[15]}$ wherein one isolate out of 80 clinical isolates was found to be VRSA with a MIC $>16 \mu \mathrm{g} / \mathrm{mL}$. In this study, all $S$. aureus isolates were subjected to susceptibility testing by the Kirby-Bauer disk diffusion method, and isolates $(n=80)$ showing a diminished zone of inhibition for vancomycin were subjected to MIC testing of vancomycin by the agar dilution method. Out of 80 isolates, 75 isolates were vancomycin sensitive (MIC $\leq 2 \mu \mathrm{g} / \mathrm{mL}$ ) and 4 isolates were VISA (MIC $4-8 \mu \mathrm{g} / \mathrm{mL}$ ). Sensitivity to linezolid among the isolates was $100 \% \cdot{ }^{[15]}$

The increasing MICs of vancomycin in $S$. aureus isolates should ring an alarm bell for prescribers, as strains with reduced susceptibility could be the indication of future strains with developed resistance. ${ }^{[15]}$

In view of the limited therapeutic options for the treatment of MRSA infections, judicious use of vancomycin, continuous surveillance for VISA and VRSA strains, and appropriate infection control practices for the prevention of spread of such strains in the hospital environment are strongly recommended..$^{[15]}$

Current treatment options for treating multidrugresistant gram-positive pathogens

The limitations of glycopeptides include poor tissue penetration, poor oral bioavailability and a narrow therapeutic window, necessitating the monitoring of serum drug levels. The problem of developing resistance among MDR strains means that treatment options within the community are becoming limited ${ }^{[4]}$ and there is an urgent need for effective novel antibiotics to target these pathogens. ${ }^{[16]}$

To overcome these shortcomings of glycopeptides, newer agents have been developed keeping in mind their efficacy against MRSA and other MDR organisms, suitable pharmacokinetic properties, tolerability and safety profile, formulation and potential for the development of resistant strains. A decision must be made on the most suitable agent for an individual patient, the site of infection and the setting for administration (healthcare or outpatient). ${ }^{[4]}$

Linezolid has been shown to be an effective agent for the treatment of nosocomial pneumonia and complicated skin and soft-tissue infections due to MDR gram-positive organisms and, as an oral agent, is ideal for treatment within the community. Newer oxazolidinones in development show similar potential. Tigecycline, ceftobiprole ( $\beta$-lactamase stable cephalosporin), ceftaroline (cephalosporin with anti-MRSA and anti-pneumococcal activity) and tompenem all have a broad spectrum of activity with excellent tissue penetration, making them ideal agents for the treatment of complicated skin and skin-structure infections. Daptomycin has been shown to be efficacious in the treatment of bacteremia and endocarditis due to MRSA and MSSA. ${ }^{[4]}$ Dalbavancin (semisynthetic lipoglycopeptide), oritavancin, telavancin (semi-synthetic vancomycin derivative), doripenem, iclaprim (new diamonipyrimidine), ranbezolid (new oxazolidinone) all show promise as agents that circumvent problems seen with vancomycin for the treatment of gram-positive infections. ${ }^{[4,16]}$

Continual research would be needed to develop new antimicrobials for MDR in gram-positive organisms. Prudent and responsible usage of these newer antibiotics is advocated to preserve their continued effectiveness in the management of difficult-to-treat infections caused by Gram-positive pathogens including MRSA, VRE, VISA, VRSA, and CoNS. ${ }^{[17]}$

\section{Emergent challenges faced by the clinicians}

The treatment of $S$. aureus infections is becoming increasingly more complicated due to the emergence of various types of antibiotic resistance. ${ }^{[13]}$ The prevalence of the $S$. aureus infections vary from place to place and so also the resistance pattern which depends upon the local 
antibiotic policy, the infection control activities, the time of the study, the number of cases which are studied and the biological characteristics of the $S$. aureus strains. ${ }^{[13]}$

The common practice in India is using single bottle of blood culture. Catheter drawn blood culture may lead to higher false positives. During the preparation of the blood drawn, there is a chance of contamination that remains a critical determinant. The volume of the blood sample taken from the patient is often inadequate. Sometime there are prolonged time gaps between sample drawn and laboratory incubation.

Clinicians specialized in infectious disease became increasingly concerned about the scarcity of antimicrobial agents available to treat MDR infections. ${ }^{[8]}$ One of the main challenges for physicians treating VRE is the intrinsic resistance to many antibiotics, including $\beta$-lactams, aminoglycosides, lincosamides, and trimethoprim-sulfamethoxazole. VRE is usually susceptible to $\beta$-lactams. Management and debridement of wounds and surgical management for source control should be performed as a first rule in the management of localized infections. ${ }^{[3]}$

Precautionary measures needs to be taken before prescribing vancomycin and the clinicians with the help of clinical microbiologist should determine the MIC of such strains to identify VISA and to prevent the emergence of vancomycin resistance. All the clinical microbiology laboratories should routinely test the MIC of vancomycin for MRSA for appropriate treatment of patients and implementation of infection control measures to prevent the spread of resistance..$^{[8]}$

Recently, the Centers for Disease Control and Prevention (CDC) issued specific recommendations intended to reduce the development and transmission of $S$. aureus with intermediate glycopeptide resistance (GISA). Vancomycin disk diffusion does not reliably identify $S$. aureus isolates with decreased susceptibility to glycopeptides. Programs to educate health care personnel about infection-control precautions against GISA should be developed, and infection-control specialists should monitor compliance with these precautions. Infection-control and laboratory personnel should implement active surveillance for GISA, particularly in populations at high risk, such as patients on dialysis and patients in whom vancomycin therapy is unsuccessful. If GISA strain is identified, prompt notification of the state health department and the CDC is critical so that epidemiologic and laboratory support can be provided. ${ }^{[8]}$
The emergence and the dissemination of resistance can be controlled by a heightened awareness of the issues, by encouraging proper personal hygiene, provision of adequate effective sewage disposal systems to prevent dissemination of the multidrug resistant bacteria from the gut, surveillance of the local bacterial population, early intervention, rigorous cross infection control measures and by the judicious use of current antimicrobial agents based on the susceptibility data. ${ }^{[13]}$

\section{CONCLUSION}

Gram-positive pathogens are a critical component of BSI and treatment of diseases caused by resistant gram-positive pathogens requires appropriate use of available antibiotics and stewardship to prolong their effectiveness. The increased prevalence of MDR strains of $S$. aureus is due to an irrational usage of broad spectrum antibiotics in the medical and the veterinary practice, high proximity to a large number of unlicensed drug vendors, poverty leading to incomplete dosage regimen of the antibiotics, antibiotic prophylaxis, high number of immunocompromised patients, the increased use of invasive procedures and devices, and inadequate infection control measures. ${ }^{[13]}$

Failure with Vancomycin occurs due to its slow bactericidal activity and it's increasing MICs. Alternative therapies should be considered where vancomycin MIC is $>1 \mu \mathrm{g} / \mathrm{mL}$ to avoid treatment failure. Early recognition of isolates resistant to the newer antibiotic agents is of paramount importance, and will allow appropriate treatment of affected patients. A Paired blood culture remains a gold standard for diagnosis of bacteremia. Appropriate, aggressive, and impeccable infection control efforts in health care facilities are vital to help prevent the spread of resistant pathogens. An attempt should be made to keep the prevalence of such resistant strains low.

\section{ACKNOWLEDGMENT}

The authors would like to thank Microbiology senior scientific officer Ms. Chongtham Geeta Chanu for in data management.

\section{REFERENCES}

1. Boucher HW. Challenges in anti-infective development in the era of bad bugs, no drugs: A regulatory perspective using the example of bloodstream infection as an indication. Clin Infectious Dis 2010;50:S4-9.

2. Thati V, Shivannavar CT, Gaddad SM. Vancomycin resistance among methicillin resistant Staphylococcus aureus isolates from intensive care units of tertiary care hospitals in Hyderabad. Indian J Med Res 2011;134:704-8.

3. Rivera AM, Boucher HW. Current concepts in antimicrobial therapy against 
select gram-positive organisms: Methicillin-resistant Staphylococcus aureus, penicillin-resistant Pneumococci, and vancomycin-resistant Enterococci. Mayo Clin Proc 2011;86:1230-42.

4. Ratnaraja NV, Hawkey PM. Current challenges in treating MRSA: What are the options? Expert Rev Anti-Infect Ther 2008;6:601-18.

5. Reddy CM, Thati V, Shivannavar CT, Gaddad SM. Vancomycin resistance among methicillin resistant Staphylococcus aureus isolates in Rayalaseema region Andhra Pradesh, South India. World J Sci Tech 2012;2:6-8.

6. Loomba PS, Tanjea J, Mishra B. Methicillin and vancomycin resistant S. aureus in hospitalized patients. J Glob Infect Dis 2010;2:275-83.

7. Tiwari HK, Sen MR. Emergence of vancomycin resistant Staphylococcus aureus (VRSA) from a tertiary care hospital from northern part of India. BMC Infect Dis 2006;6:156.

8. Sharma P, Vishwanath G. Study of vancomycin susceptibility in methicillin-resistant Staphylococcus aureus isolated from clinical samples. Ann Trop Med Public Health 2012;5:178-80.

9. Woodford N, Livermore DM. Infections caused by Gram-positive bacteria: A review of the global challenge. J Infect 2009;59 Suppl 1:S4-16.

10. Appelbaum PC. MRSA--the tip of the iceberg. Clin Microbiol Infect 2006;12 Suppl 2:3-10.

11. Tarai B, Das P, Kumar D, Budhiraja S. Comparative evaluation of paired blood culture (aerobic/aerobic) and single blood culture, along with clinical importance in catheter verses peripheral line at a tertiary care hospital. Int J Med Microbiol 2012:30:187-92.

12. Sharma A, Kutty CV, Sabharwal U, Rathee S, Mohan H. Evaluation of Sepsis screen for diagnosis of neonatal septicemia. Indian J Pediatr 1993;60:559-63.

13. Dhanalakshmi TA, Umapathy BL, Mohan DR. Prevalence of Methicillin, vancomycin and multidrug resistance among Staphylococcus aureus. J Clin Diagn Res 2012;6:974-7.

14. Nicolaou KC, Boddy CN, Bräse S, Winssinger N. Chemistry, biology, and medicine of the glycopeptide antibiotics. Angew Chem Int Ed 1999;38:2096-152.

15. Ramana BV, Chaudhury A. Rising incidence of high MIC for vancomycin among Staphylococcus aureus strains at a tertiary care hospital in South India. J Pharm Bioallied Sci 2012;4:173.

16. Banwana K, Senokb AC, Rotimic VO. Antibiotic therapeutic options for infections caused by drug-resistant Gram-positive cocci. J Infect Public Health 2009;2:62-73.

17. Schwarz S, Silley P, Simjee S, Woodford N, van Duijkeren E, Johnson AP, et al. Editorial: Assessing the antimicrobial susceptibility of bacteria obtained from animals. J Antimicrob Chemother 2010;65:601-4.

How to cite this article: Tarai B, Das $P$, Kumar D. Recurrent challenges for clinicians: emergence of methicillin-resistant Staphylococcus aureus, vancomycin resistance, and current treatment options. J Lab Physicians 2013;5:71-8.

Source of Support: Max Super Speciality Hospital. Conflict of Interest: None declared.

\section{“QUICK RESPONSE CODE” LINK FOR FULL TEXT ARTICLES}

The journal issue has a unique new feature for reaching to the journal's website without typing a single letter. Each article on its first page has a "Quick Response Code". Using any mobile or other hand-held device with camera and GPRS/other internet source, one can reach to the full text of that particular article on the journal's website. Start a QR-code reading software (see list of free applications from http://tinyurl.com/yzlh2tc) and point the camera to the QR-code printed in the journal. It will automatically take you to the HTML full text of that article. One can also use a desktop or laptop with web camera for similar functionality. See http://tinyurl.com/2bw7fn3 or http://tinyurl.com/3ysr3me for the free applications. 